\title{
Application of DDG-3300K Liver Reserve Function Analyzer and Relevant Nursing Procedures at the Department of Infectious Diseases
}

\author{
Lihua Zheng1, Cheng Hu², Yubao Zheng1, Qiong Shu1', Xiang Yin¹, Hongmei Pan1, Haotong Xu1, \\ Beiqian $\mathrm{Wu}^{1}$, Ying Liu ${ }^{*}$
}

${ }^{1}$ Department of Infectious Diseases, The Third Affiliated Hospital of Sun Yat-sen University, Guangzhou, China

${ }^{2}$ Department of Urology, The Third Affiliated Hospital of Sun Yat-sen University, Guangzhou, China

Email: *liuy35@mail.sysu.edu.cn

How to cite this paper: Zheng, L.H., Hu, C., Zheng, Y.B., Shu, Q., Yin, X., Pan, H.M., $\mathrm{Xu}$, H.T., Wu, B.Q. and Liu, Y. (2019) Application of DDG-3300K Liver Reserve Function Analyzer and Relevant Nursing Procedures at the Department of Infectious Diseases. Open Journal of Nursing, 9, 120-124.

https://doi.org/10.4236/ojn.2019.92011

Received: January 10, 2019

Accepted: February 15, 2019

Published: February 18, 2019

Copyright $\odot 2019$ by author(s) and Scientific Research Publishing Inc. This work is licensed under the Creative Commons Attribution International License (CC BY 4.0).

http://creativecommons.org/licenses/by/4.0/

\begin{abstract}
Objective: To discuss some key points about nursing in the use of DDG-3300K liver reserve function analyzer in patients at the department of infectious diseases. Method: DDG-3300K liver reserve function analyzer was applied to 5464 patients at the department of infectious diseases. The reasons for failed detection and complications related to the detection were analyzed, and the measures for improving the nursing procedures were proposed. Result: Among the 5464 patients, the detections were successful at the first attempt in 5458 patients; 2 patients had leakage of liquid; 2 patients were poorly prepared, and 1 case failed because of mistaken selection of $\mathrm{CO}$ mode, which led to adverse drug reactions; 1 case did not finish the detection due to anaphylactic shock; 8 patients had nausea and 6 patients had skin rash on the four limbs and torso during the detection. Conclusion: It is necessary to formulate the nursing procedures for the use of DDG-3300K liver reserve function analyzer. Moreover, preparatory work, health education, refined nursing procedures and skillful operations are closely related to the success rate and accuracy of the detection.
\end{abstract}

\section{Keywords}

DDG-3300K Liver Reserve Function Analyzer, Liver Function Reserve, Indocyanine Green (ICG), Nursing

\section{Introduction}

Indocyanine green (ICG) clearance test and Child-Pugh classification are the 
two commonly used methods to assess liver parenchyma function in clinic [1]. ICG clearance test is easy to perform with the use of DDG-3300K liver reserve function analyzer, causing small invasiveness and less pain and receiving little interferences [2].

The present study aimed to discuss the key points of nursing for patients receiving liver reserve function test using DDG-3300K liver reserve function analyzer. To this end, from July 2016 to December 2018, 5464 patients receiving tests with the DDG-3300K liver reserve function analyzer were recruited. The experience of applying DDG-3300K liver reserve function analyzer to these 5464 was reviewed.

\section{Materials and Method}

\subsection{Baseline Data}

There were 4476 males and 988 females, aged 9 - 78 years old. The patients had hepatic failure, cirrhosis, viral hepatitis, space-occupying lesions of the liver and autoimmune hepatitis.

\subsection{Method}

1) Equipment and reagent: DDG-3300K liver reserve function analyzer (Nihon Kohden, with host machine, display, printer and nasal probe), and ICG (Dandong Yichang Pharmaceutical Co., Ltd., $2.5 \mathrm{mg} /$ bottle).

2) Detection method: The patients were fasted from food and water for $2-4 \mathrm{~h}$. They were told to defecate and urinate before detection, and took a supine position during detection. Mobile phones and other wireless communications in the ward were turned off during detection. The host machine, display and printer of the DDG-3300K liver reserve function analyzer were turned on, and the nasal probe was attached to one side of the nose wing. The connection line was immobilized to the forehead with adhesive tape. BV/K mode was selected on the MENU, and patient information was input: hemoglobin level ( $\mathrm{g} / \mathrm{dl}$ ), actual dose of ICG $(\mathrm{mg})$, height $(\mathrm{cm})$ and weight $(\mathrm{kg})$. The elbow vein was punctured and ICG diluted to $5 \mathrm{mg} / \mathrm{ml}$ was injected at a constant rate within $10 \mathrm{~s}$. The blood level of ICG was continuously monitored with the nasal probe.

\section{Results}

Among the 5464 patients, the detections were successful at the first attempt in 5458 patients; 2 patients had leakage of liquid, 2 patients were poorly prepared, and 1 case failed because of mistaken selection of $\mathrm{CO}$ mode, which led to adverse drug reactions; 1 case did not finish the detection due to anaphylactic shock; 8 patients had nausea and 6 patients had skin rash on the four limbs and torso during the detection.

\section{Discussion}

Assessment method for liver function is divided into static and dynamic detec- 
tion. Static detection is more commonly used and usually performed through liver biochemical test. However, the abnormal levels of the liver biochemical indicators are not necessarily consistent with the severity of liver diseases. ICG clearance test is a dynamic detection method that has found the most extensive applications. ICG is a non-toxic pigment and its intake by the liver cells is selective after intravenous injection. ICG is converted intracellularly and excreted across the membrane along with the bile. Preparatory work, health education and skilled manipulation are crucial for the success and accuracy of detection.

\subsection{Safety Nursing}

1) Risk, harm and countermeasures for drug allergy: ICG may cause anaphylactic shock, nausea and vomiting. Emergency drugs and other materials needed for emergency were ready during the detection. History of drug allergy was carefully inquired. The detection was contraindicated for those with iodine hypersensitivity, or should be performed with caution in those with allergic constitution [3]. Adverse reactions were closely observed during detection, including dizziness, numbness in the mouth, conjunctival congestion and edema, palpitation, chest tightness, nausea \& vomiting, and pain at the injection site. The venous access was preserved if the above symptoms happened, and reporting was immediately made to the physicians for proper treatment. In the present study, one patient had dizziness and coldness in the four limbs after the injection, with blood pressure decreasing until no longer detectable and pulse rate 52 beats/min. For this patient, anti-shock treatment was immediately given as instructed, with intravenous injection of $10 \mathrm{ml}$ of $0.9 \%$ normal saline plus $5 \mathrm{mg}$ dexamethasone, intramuscular injection of $0.05 \mathrm{mg}$ adrenaline, use of dopamine for blood pressure regulation and Ringer's solution for dilatancy. The vital signs were closely monitored, and the patient gradually recovered consciousness $20 \mathrm{~min}$ later with warmness returning to the four limbs, blood pressure up to $116 / 75$ $\mathrm{mmHg}$ and heart rate 77 beats $/ \mathrm{min}$. Then dopamine was discontinued. A total of 8 patients had nausea, and 6 patients skin rash in the four limbs and torso. They were given intravenous injection of $10 \mathrm{ml} 10 \%$ glucose injection $+10 \mathrm{~mL} 10 \%$ calcium gluconate injection. The vital signs were closely monitored, and all associated symptoms disappeared in these patients after proper treatment.

2) Importance and measures of vascular protection: Vein indwelling needles for infusion in the forearm were used in two patients. As the needles were already indwelled for $72 \mathrm{~h}$, the vascular permeability increased, resulting in large pressure and resistance upon drug injection. Therefore, a small amount of the liquid leaked into the subcutaneous tissues, and the detection failed because the blood level of the drug and injection rate of the drug did not meet the standard. The liquid leak also led to the green color of the skin. The patient had local pain at the injection site and the indwelling needle was removed. Sanyrene was applied t.i.d to the local skin. ICG is a cyanine dye used in medical diagnostics and will not cause skin pigmentation. The skin color turned normal on the next day and the pain disappeared. 
3) Importance and standard operation of drug dilution: ICG was completely dissolved in the sterile water for injection. No other solutions such as normal saline were allowed to use. A syringe could be used for repeated drawing and injection during the dissolution. The diluted drug was not suitable for use if there was any undissolved drug on the glass wall. It was important to prevent nausea, fever and shock caused by ensuring complete drug dissolution.

\subsection{Measures to Avoid Cross-Infection}

The nasal probe was disinfected with cotton swab and soft cloth soaked in $75 \%$ alcohol before and after use. Then the probe was fully dried to prevent cross-infection. No other disinfectants were allowed to use, otherwise the probe would age and be affected in precision. The nasal probe was not applicable for those with defects in the skin of the nose or nasal mucosa, and finger probe was used instead.

\subsection{Eliminating the Interfering Factors}

1) Preparatory work: It was ensured that the pulse value, pulse rate and blood oxygen were stable. At the early stage of detection, LOW QUALITY was shown on the display due to cold weather and cold nose wings in winter. This indicated poor warming effect and the detection failed. After that, if $\mathrm{Hb}<6 \mathrm{~g} / \mathrm{dL}$ (indicating poor blood circulation in the brain and coldness of the face) and the pulse value was below grade 2, warming measures were taken by applying the towel soaked in hot water to the face. The detection did not restart until the pulse value was above grade 2. By using this method, the detections did not fail again in any patients. For the above patient in which the first detection failed, the second detection was performed $1 \mathrm{~h}$ later and was successful. The heart rate was below 50 beats/min in two patients. LOW QULITY was shown on the display as the heart rate slowed down when these two patients entered the quiescent state. They were told to move the four limbs to promote systemic blood circulation and to avoid excessively low heart rate. Thus the warning disappeared and the detections were successful.

2) Cooperations from the patients and relatives: The patients were informed of the significance, safety, basic procedures and precautions of ICG clearance test before detection, so as to sooth their anxiety and help them keep quiescent and be cooperative. Tightening nose and frowning might induce failure of detection [4]. During the detection, the patients were not allowed to shake head, talk or flip the nose wings to avoid dislocation of the nasal probe or failure of detection. If there were such symptoms as cough and sneezing, symptomatic treatments were needed before the detection. Intranasal uncleanliness would affect the connection of the infrared sensor of the nasal probe, leading to failure of detection. If there was plenty of nasal secretion, the nasal cavity should be first cleaned before detection [5]. It was ensured that the nasal probe was accurately positioned and properly fixed before ICG injection and that the injection pathway was unobstructed. The drug was gently injected at a constant rate within $10 \mathrm{~s}$ while watching the reactions of the patients. 
3) Environmental preparation and emergency handling: The environment was kept in order and the mobile phones and wireless devices were turned off or switched to the airplane mode. The ward was kept quiet. The pulses were felt to be irregular during detection in 1 patient, and the physician was informed of this situation. Later this patient received bedside ECG after liver reserve function analysis, which indicated atrial fibrillation. This patient was transferred to the department of cardiology for further treatment after consultation.

4) Development and implementation of standardized procedures: The procedures were performed in strict accordance to the standards. Preparatory work and health education were enhanced, as they were equally important as refined nursing and skillful operations in ensuring the success and accuracy of the detection.

\section{Conclusion}

The ICG clearance test measures the real-time physiological functions of the liver through multiple steps and in a comprehensive manner. It is an important method of liver reserve function detection. The nurses must be fully prepared before detection and carry out health education as required. These are equally important as standardized operations and quality control for the success and accuracy of the liver reserve function analysis.

\section{Acknowledgements and Funding}

This work was funded by the Natural Science Foundation of Guangdong Province (NO. 2017A030313620) and the National Natural Science Foundation of China (NO. 81802536).

\section{Conflicts of Interest}

The authors declare no conflicts of interest regarding the publication of this paper.

\section{References}

[1] Li, Z. and Zhu, J.Y. (2017) An Interpretation of Diagnosis, Management, and Treatment of Hepatocellular Carcinoma. Journal of Clinical Hepatology, No. 9, 1655-1657.

[2] Lin, Z. and Shi, T. (2018) One Case of ICG-Induced Allergy. Chinese Journal of Pharmacovigilance, No. 1, 63-64.

[3] Wang, X.L., Xu, M.F., Chen, H., et al. (2011) Some Experience in the Nursing for ICG-Induced Adverse Reaction in Liver Reserve Function Detection. Strait Pharmaceutical Journal, No. 5, 262-263.

[4] Huang, G.Y. (2011) Significance of Nursing Intervention in Liver Reserve Function Analysis Using DDG Analyzer. Chinese Journal of Medical Device, No. 10, 79-80.

[5] Jin, R.Y. (2013) Reasons and Nursing Countermeasures for the Failure of Liver Reserve Function Detection. Journal of Nurses Training, No. 13, 1242-1243. 